\title{
Global Dynamics of the Chaotic Disk Dynamo System Driven by Noise
}

\author{
Chunsheng Feng $\mathbb{D},{ }^{1}$ Lijie Li $\mathbb{D},{ }^{2}$ Yongjian Liu $\left(\mathbb{D},{ }^{2}\right.$ and Zhouchao Wei $\mathbb{D}^{3}$ \\ ${ }^{1}$ School of Mathematics and Computational Science, Xiangtan University, Xiangtan 411105, China \\ ${ }^{2}$ Guangxi Colleges and Universities Key Laboratory of Complex System Optimization and Big Data Processing, \\ Yulin Normal University, Yulin 537000, China \\ ${ }^{3}$ School of Mathematics and Physics, China University of Geosciences, Wuhan 430074, China \\ Correspondence should be addressed to Lijie Li; lilijie1219@126.com and Zhouchao Wei; weizhouchao@163.com
}

Received 24 December 2019; Revised 14 February 2020; Accepted 28 February 2020; Published 26 March 2020

Academic Editor: Hassan Zargarzadeh

Copyright (C) 2020 Chunsheng Feng et al. This is an open access article distributed under the Creative Commons Attribution License, which permits unrestricted use, distribution, and reproduction in any medium, provided the original work is properly cited.

\begin{abstract}
The disk dynamo system, which is capable of chaotic behaviours, is obtained experimentally from two disk dynamos connected together. It models the geomagnetic field and is used to explain the reversals in its polarity. Actually, the parameters of the chaotic systems exhibit random fluctuation to a greater or lesser extent, which can carefully describe the disturbance made by environmental noise. The global dynamics of the chaotic disk dynamo system with random fluctuating parameters are concerned, and some new results are presented. Based on the generalized Lyapunov function, the globally attractive and positive invariant set is given, including a two-dimensional parabolic ultimate boundary and a four-dimensional ellipsoidal ultimate boundary. Furthermore, a set of sufficient conditions is derived for all solutions of the stochastic disk dynamo system being global convergent to the equilibrium point. Finally, numerical simulations are presented for verification.
\end{abstract}

\section{Introduction}

The magnetic field has reversed its polarity many times along geological history [1]. To geophysics, their fundamental goal is a coherent understanding of the structure and dynamics of the Earth's interior. A number of investigators worked hard in order to establish the state of the Earth's dynamo. Bullard studied a disk dynamo with the intention of discussing possible analogies between them and those of a homogeneous dynamo which is supposed to be the origin of the magnetic field of the Earth and other celestial bodies. Before long, Japanese geophysicist Rikitake [2] found that reversals of electric current generated by a circuit can often occur even in a very simple system such as the one with two disk dynamos. The behaviour of the system is far different from that of the single disk dynamo, which never has a reversal of the electric current. Then, a simple mechanical model used to study the reversals of the Earth's magnetic field is a two-disc dynamo system idealized by Rikitake. The model consists of two identical single Faraday-disk dynamos of the Bullard type coupled together. For simplicity, we denote the angular velocities of their rotors by $x_{3}$ and $x_{4}$ and the currents generated by $x_{1}$ and $x_{2}$, respectively. Then, with appropriate normalization of variables, the dynamical equations can be described by the following set of ordinary differential equations $[3,4]$ :

$$
\left\{\begin{array}{l}
\dot{x}_{1}=-\mu_{1} x_{1}+x_{2} x_{3}, \\
\dot{x}_{2}=-\mu_{2} x_{2}+x_{1} x_{4}, \\
\dot{x}_{3}=q_{1}-\epsilon_{1} x_{3}-x_{1} x_{2}, \\
\dot{x}_{4}=q_{2}-\epsilon_{2} x_{4}-x_{1} x_{2} .
\end{array}\right.
$$

where $q_{1}$ and $q_{2}$ are the torques applied to the rotors and $\mu_{1}$, $\mu_{2}, \epsilon_{1}$, and $\epsilon_{2}$ are the positive constants representing dissipative effects of the disk dynamo system. Rather, from the physical meaning of the equation, the parameters $\mu$ and $\epsilon$ 
represent the power consumption and mechanical damping dissipation of disk dynamo, respectively. When the parameters are $\mu_{1}=3, \mu_{2}=1, \epsilon_{1}=0.1, \epsilon_{2}=0.2, q_{1}=3$, and $q_{2}=1$ for initial states $\left(x_{1}(0), x_{2}(0), x_{3}(0), x_{4}(0)\right)=(2.2,2.0,10.5$, $20)$, the numerical simulation shows that the corresponding Lyapunov exponents are $0.28,0,-0.10$, and -4.47 . There exists one positive Lyapunov exponent suggest that system (1) has a chaotic attractor. The chaotic attractor's projections in the coordinate planes $x_{1}-x_{2}-x_{3}$ and $x_{2}-x_{3}-x_{4}$ are shown in Figure 1.

One the one hand, since the Lorenz system [5] was presented, there is a huge volume of the literature devoted to the studies of the Lorenz system and other classical chaotic systems, which are closely related but not topologically equivalent to the Lorenz system, such as Chen system [6], Lü system [7], and Yang system [8]. In a sense defined by Vaněček and Čelikovský $[9,10]$, the Chen system is a dual system to the Lorenz system and the Lü system and Yang system represent a transition between the Lorenz and the Chen systems. For the Lorenz family system, mathematicians, physicists, and engineers from various fields have studied the characteristics of systems, bifurcations, routes to chaos, essence of chaos, and chaos synchronization. By ignoring mechanical damping dissipation that parameters $\epsilon_{1}=\epsilon_{2}=0$ and setting $q_{1}=q_{2}=1$ and $\mu_{1}=\mu_{2}=\mu$, we can write $x_{3}=z$ and $x_{4}=z-\alpha$, where $\alpha$ is a constant of the motion. Finally, coupled dynamos (1) can be written in the following simple form [11]:

$$
\left\{\begin{array}{l}
\dot{x}_{1}=-\mu x_{1}+x_{2} z \\
\dot{x}_{2}=-\mu x_{2}+x_{1}(z-\alpha) \\
\dot{z}=1-x_{1} x_{2}
\end{array}\right.
$$

System (2) has a three-dimensional attractor similar to the Lorenz attractor although both systems are obviously not topologically equivalent [11]. The chaotic behavior and other properties, synchronization and control of the disk dynamo system and disk dynamo-like chaotic systems (2), were extensively studied (see, for instance, [11-16] and their references).

On the other hand, Arnold [17] has pointed out that the parameters in the chaotic systems exhibit random fluctuation to a greater or lesser extent due to various environmental noise. Scholars usually estimate them by average values plus some error terms [18]. In general, by the wellknown central limit theorem, the error terms follow normal distributions. For the best incorporate (natural) randomness into the mathematical description of the phenomena and to provide a more accurate description of it, we model the stochastic disk dynamo system by replacing the parameters $\mu_{1}, \quad \mu_{2}, \epsilon_{1}, \epsilon_{2}, \quad q_{1}, \quad$ and $q_{2}$ by $\mu_{1} \longrightarrow \mu_{1}+\sigma_{1} \mathrm{~d} W(t)$, $\mu_{2} \longrightarrow \mu_{2}+\sigma_{2} \mathrm{~d} W(t), \quad \epsilon_{1} \longrightarrow \epsilon_{1}+\sigma_{3} \mathrm{~d} W(t), \quad \epsilon_{2} \longrightarrow \epsilon_{2}+$ $\sigma_{4} \mathrm{~d} W(t), q_{100} \longrightarrow q_{1}+q_{10} \mathrm{~d} W(t)$, and $q_{2} \longrightarrow q_{2}+q_{20} \mathrm{~d} W(t)$, where $W(t)$ are the mutually independent Brownian motions. Then, one gets the following system of stochastic differential equations:

$$
\left\{\begin{array}{l}
\mathrm{d} x_{1}(t)=\left(-\mu_{1} x_{1}+x_{2} x_{3}\right) \mathrm{d} t+\sigma_{1} x_{1} \mathrm{~d} W(t), \\
\mathrm{d} x_{2}(t)=\left(-\mu_{2} x_{2}+x_{1} x_{4}\right) \mathrm{d} t+\sigma_{2} x_{2} \mathrm{~d} W(t), \\
\mathrm{d} x_{3}(t)=\left(q_{1}-\epsilon_{1} x_{3}-x_{1} x_{2}\right) \mathrm{d} t+\left(\sigma_{3} x_{3}+q_{10}\right) \mathrm{d} W(t), \\
\mathrm{d} x_{4}(t)=\left(q_{2}-\epsilon_{2} x_{4}-x_{1} x_{2}\right) \mathrm{d} t+\left(\sigma_{4} x_{4}+q_{20}\right) \mathrm{d} W(t) .
\end{array}\right.
$$

To illustrate the stochastic effects clearly, we performed simulations for the corresponding stochastic case of Figure 1. The corresponding stochastic case uses the same parameters and initial values. Let $\mu_{1}=3, \mu_{2}=1, \epsilon_{1}=0.1$, $\epsilon_{2}=0.2, q_{1}=3$, and $q_{2}=1$ and initial states $\left(x_{1}(0), x_{2}(0), x_{3}(0)\right.$, $\left.x_{4}(0)\right)=(2.2,2.0,10.5,20)$ additionally have the perturbed parameters $\sigma_{1}=0.1, \sigma_{2}=0.1, \sigma_{3}=0.01, \sigma_{4}=0.01, q_{10}=0.1$, and $q_{20}=0.1$. The projections in the coordinate planes $x_{1}-x_{2}-x_{3}$ and $x_{2}-x_{3}-x_{4}$ are shown in Figure 2. Comparing Figures 1 and 2, we can see the difference between the deterministic case and stochastic case. Actually, the behavior of system will change even if the parameters suffer small perturbation. Suppose that other parameters remain unchanged and only $\epsilon_{2}$ change; let $\mu_{1}=3, \mu_{2}=1, \epsilon_{1}=0.1$, $\epsilon_{2}=0.2, q_{1}=3$, and $q_{2}=1$, initial states $\left(x_{1}(0), x_{2}(0), x_{3}(0)\right.$, $\left.x_{4}(0)\right)=(2.2,2.0,10.5,20)$, and the perturbed parameters $\sigma_{1}=0, \sigma_{2}=0, \sigma_{3}=0, \sigma_{4}=0.01, q_{10}=0$, and $q_{20}=0$. Time series $x_{1}$ diagram of the deterministic case and stochastic case are shown in Figure 3.

Chaos synchronization is a very important topic in chaos theory. Enormous research activities have been carried out in chaos synchronization by many researchers from different disciplines, and lots of successful experiments have been reported. Many scholars, by using capacitor coupling [19], induction coil coupling [20], and resistance coupling [21] to realize the synchronization of chaotic systems, have obtained good results. In chaotic synchronization, the boundedness of the system is a very important prerequisite. In fact, ultimate boundedness of chaotic dynamical systems is always one of the fundamental concepts in dynamical systems. This plays an important role in investigating the stability of the equilibrium, estimating the Lyapunov dimension of attractors and the Hausdorff dimension of attractors, the existence of periodic solutions, chaos control, and chaos synchronization. Technically, to locate and estimate the relative position of the attractor is a difficult work even in a deterministic system [22-26]. For the deterministic system, $\mathrm{Yu}$ and Liao [27] give the concept of the exponential attractive set and estimate the globally attractive and positive invariant set of the typical Lorenz system. For the stochastic system, some results of the estimation global attractive set have also been obtained, for the stochastic Lorenz-Stenflo system [18], the stochastic Lorenz-Haken system [28], the stochastic Lorenz-84 system [29], the stochastic Lorenz system family [30], the stochastic Rabinovich system $[31,32]$, and other stochastic systems [33, 34].

In this paper, by using a technique combining the generalized Lyapunov function theory and optimization, globally exponential attractive set and a four-dimensional 


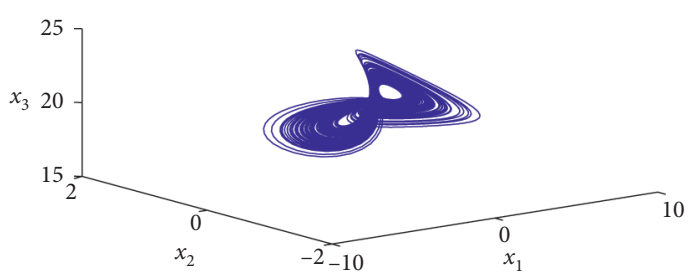

(a)

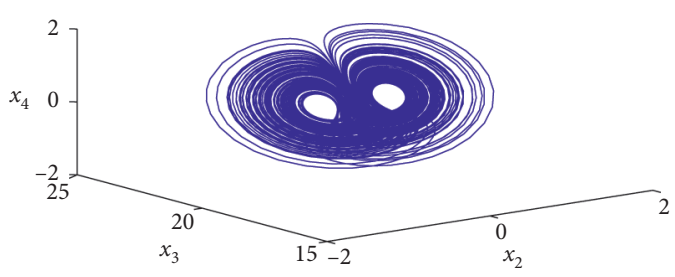

(b)

FIGURE 1: The disk dynamo system exhibits chaotic behavior of system parameters $\mu_{1}=3, \mu_{2}=1, \epsilon_{1}=0.1, \epsilon_{2}=0.2, q_{1}=3$, and $q_{2}=1$ and initial values $\left(x_{0}, y_{0}, z_{0}, u_{0}\right)=(2.2,2.0,10.5,20)$.

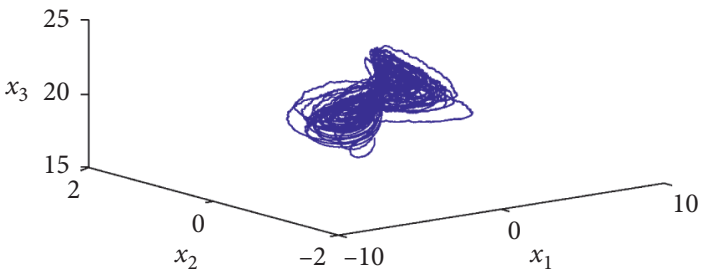

(a)

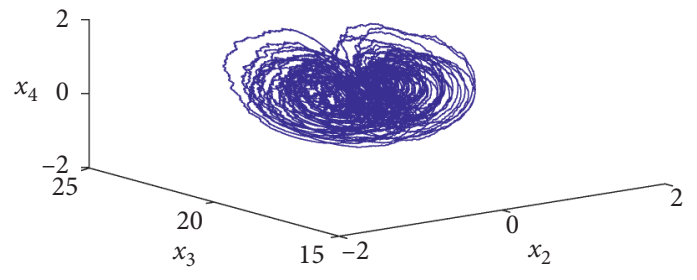

(b)

Figure 2: Simulated phase portraits of stochastic disk dynamo system (3) with parameters $\mu_{1}=3, \mu_{2}=1, \epsilon_{1}=0.1, \epsilon_{2}=0.2, q_{1}=3, q_{2}=1$, $\sigma_{1}=0.1, \sigma_{2}=0.1, \sigma_{3}=0.01, \sigma_{4}=0.01, q_{10}=0.1, q_{20}=0.1$ and initial values $\left(x_{0}, y_{0}, z_{0}, u_{0}\right)=(2.2,2.0,10.5,20)$.

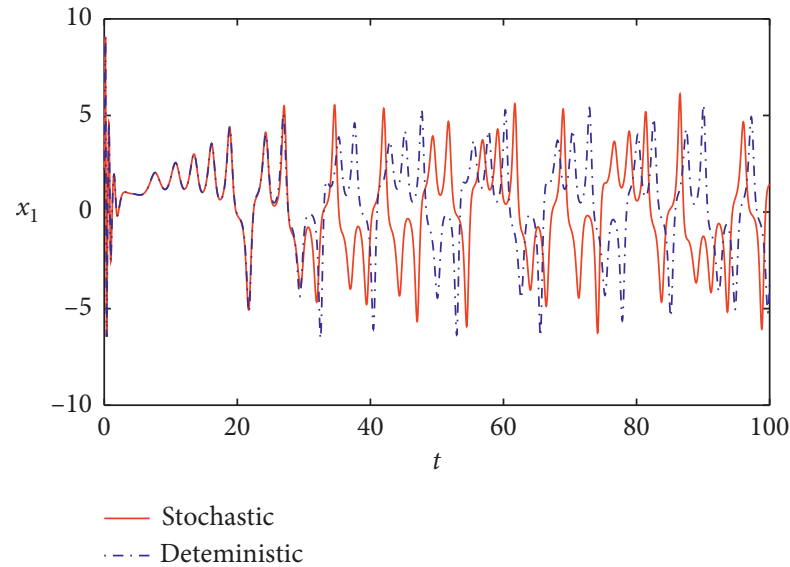

Figure 3: Time series diagrams of the deterministic and stochastic disk dynamo system with parameters $\mu_{1}=3, \mu_{2}=1, \epsilon_{1}=0.1, \epsilon_{2}=0.2$, $q_{1}=3, q_{2}=1, \sigma_{1}=0, \sigma_{2}=0, \sigma_{3}=0, \sigma_{4}=0$ (deterministic case), $\sigma_{4}=0.01$ (stochastic case), $q_{10}=0, q_{20}=0$, and initial values $\left(x_{0}, y_{0}\right.$, $\left.z_{0}, u_{0}\right)=(2.2,2.0,10.5,20)$.

ellipsoidal ultimate bound are derived, which can help us to locate the relative position of the attractor. The two-dimensional parabolic ultimate bound is also established. And numerical results to estimate the ultimate bound are also presented for verification. We hope that the investigation of this paper can help understanding the rich dynamic of the stochastic disk dynamo system and offer some enlightenments for the study of the reversals of the Earth's magnetic field.

This paper is organized as follows. In Section 2, the cylindrical bound of stochastic disk dynamo system (3) is presented. In Section 3, globally exponential attractive set and positive invariant set of the system are derived. In Section 4, the stochastic stability of system (3) is studied. In each section, we also give corresponding numerical results, respectively. The conclusions are given in Section 5 .

\section{Cylindrical Bound}

Theorem 1. Let $l_{13}=\min \left\{2\left(\mu_{1}-(1 / 2) \sigma_{1}^{2}\right), \epsilon_{1}-(1 / 2) \sigma_{3}^{2}\right\}$ and $l_{24}=\min \left\{2\left(\mu_{2}-(1 / 2) \sigma_{3}^{2}\right), \epsilon_{2}-(1 / 2) \sigma_{4}^{2}\right\}$. Suppose that the parameters $2 \mu_{1}>\sigma_{1}^{2}, 2 \mu_{2}>\sigma_{2}^{2}, 2 \epsilon_{1}>\sigma_{3}^{2}$, and $2 \epsilon_{2}>\sigma_{4}^{2}$, $\sigma_{1} \geq 0(i=1,2,3,4)$. Then, the set $\Omega$ is the bound for system (3), in the sense that system (3) is the cylindrical bound, where

$$
\begin{aligned}
\Omega= & \left\{X \mid E\left[x_{1}^{2}+x_{3}^{2}\right] \leq \frac{\left(q_{1}+q_{10} \sigma_{3}\right)^{2}}{l_{13}\left(\epsilon_{1}-(1 / 2) \sigma_{3}^{2}\right)}+\frac{q_{10}^{2}}{l_{13}}, E\left[x_{2}^{2}+x_{4}^{2}\right]\right. \\
& \left.\leq \frac{\left(q_{2}+q_{20} \sigma_{4}\right)^{2}}{l_{24}\left(\epsilon_{2}-(1 / 2) \sigma_{4}^{2}\right)}+\frac{q_{20}^{2}}{l_{24}}\right\} .
\end{aligned}
$$

Proof

Step 1. Construct a positive definite and radically unbounded Lyapunov function on $\mathbb{R}^{2}$ :

$$
V_{13}\left(x_{1}, x_{3}\right)=\frac{1}{2}\left(x_{1}^{2}+x_{3}^{2}\right) \text {. }
$$

Applying Itô's formula, one has 


$$
\begin{aligned}
\mathrm{d} V_{13}= & {\left[-\mu_{1} x_{1}^{2}-\epsilon_{1} x_{3}^{2}+q_{1} x_{3}+\frac{1}{2}\left(\sigma_{1}^{2} x_{1}^{2}+\left(\sigma_{3} x_{3}+q_{10}\right)^{2}\right)\right] \mathrm{d} t } \\
& +\left(\sigma_{1} x_{1}^{2}+\sigma_{3} x_{3}^{2}+q_{10} x_{3}\right) \mathrm{d} W(t) \\
\leq & {\left[-\left(\mu_{1}-\frac{1}{2} \sigma_{1}^{2}\right) x_{1}^{2}-\frac{1}{2}\left(\epsilon_{1}-\frac{1}{2} \sigma_{3}^{2}\right) x_{3}^{2}+L_{13}\right] \mathrm{d} t } \\
& +\left(\sigma_{1} x_{1}^{2}+2 \sigma_{3} x_{3}^{2}+\frac{q_{10}^{2}}{4 \sigma_{3}}\right) \mathrm{d} W(t) \\
\leq & \left(l_{13} V_{13}+L_{13}\right) \mathrm{d} t+\left(l_{3} V_{13}+\frac{q_{10}^{2}}{4 \sigma_{3}}\right) \mathrm{d} W(t),
\end{aligned}
$$

where

$$
\begin{aligned}
l_{13} & =\min \left\{2\left(\mu_{1}-\frac{1}{2} \sigma_{1}^{2}\right), \quad \epsilon_{1}-\frac{1}{2} \sigma_{3}^{2}\right\}, \\
l_{3} & =\max \left\{2 \sigma_{1}, 4 \sigma_{3}\right\} \\
L_{13} & =\frac{\left(q_{1}+q_{10} \sigma_{3}\right)^{2}}{2\left(\mu_{1}-(1 / 2) \sigma_{1}^{2}\right)}+\frac{1}{2} q_{10}^{2} .
\end{aligned}
$$

Similar to the proof of Theorem 1, we can obtain $E\left[V_{13}-\frac{L_{13}}{l_{13}}\right] \leq\left[V_{13}\left(x_{1}\left(t_{0}\right), x_{3}\left(t_{0}\right)\right)-\frac{L_{13}}{l_{13}}\right] \exp \left\{-l_{13}\left(t-t_{0}\right)\right\}$.

Therefore, one has $\lim _{t \longrightarrow+\infty} E V_{13} \leq\left(L_{13} / l_{13}\right)$; that is to say, the following inequality holds as $t \longrightarrow+\infty$ :

$$
E\left[x_{1}^{2}+x_{3}^{2}\right] \leq \frac{2 L_{13}}{l_{13}}
$$

Step 2. Construct a positive definite and radically unbounded Lyapunov function on $\mathbb{R}^{2}$ :

$$
V_{24}\left(x_{2}, x_{4}\right)=\frac{1}{2}\left(x_{2}^{2}+x_{4}^{2}\right) \text {. }
$$

Similar to the proof of Step 1, we can obtain

$$
\mathrm{d} V_{24} \leq\left(l_{24} V_{24}+L_{24}\right) \mathrm{d} t+l_{4} V_{24} \mathrm{~d} W(t),
$$

where

$$
\begin{aligned}
l_{24} & =\min \left\{2\left(\mu_{2}-\frac{1}{2} \sigma_{3}^{2}\right), \epsilon_{2}-\frac{1}{2} \sigma_{4}^{2}\right\} \\
l_{1} & =\max \left\{2 \sigma_{3}, 4 \sigma_{4}\right\} \\
L_{24} & =\frac{\left(q_{2}+q_{20} \sigma_{4}\right)^{2}}{2\left(\epsilon_{2}-(1 / 2) \sigma_{4}^{2}\right)}+\frac{1}{2} q_{20}^{2} .
\end{aligned}
$$

Therefore, the following inequality holds as $t \longrightarrow+\infty$ :

$$
E\left[x_{2}^{2}+x_{4}^{2}\right] \leq \frac{2 L_{24}}{l_{24}}
$$

By Step 1 and Step 2, system (3) is the cylindrical bound.
Remark 1. Let $\sigma_{1}=\sigma_{2}=\sigma_{3}=\sigma_{4}=0$; then system (3) is deterministic. Theorem 2 contains the results given in [12] as special cases.

Let $\mu_{1}=3, \quad \mu_{2}=1, \quad \epsilon_{1}=0.1, \quad \epsilon_{2}=0.2, \quad q_{1}=3, \quad q_{2}=1$, $\sigma_{1}=\sigma_{2}=0.1, \sigma_{3}=\sigma_{4}=0.01$, and $q_{10}=q_{20}=0.1$, and initial values $\left(x_{0}, y_{0}, z_{0}, u_{0}\right)=(2.2,2,2.5,3)$. Calculate $l_{13}=0.099950$ and $L_{24}=0.199950$. We give the following estimate of the ultimate boundary:

$$
\Omega=\left\{X \mid E\left[x_{1}^{2}+x_{3}^{2}\right] \leq 901.601426, E\left[x_{2}^{2}+x_{4}^{2}\right] \leq 25.112567\right\} .
$$

The corresponding projections of exponentially attractive sets are shown in Figure 4.

And we also have the following results:

$$
\begin{aligned}
& \left|\mathrm{E} x_{1}\right| \leq 30.026679 \\
& \left|\mathrm{E} x_{2}\right| \leq 5.011244 \\
& \left|\mathrm{E} x_{3}\right| \leq 30.026679 \\
& \left|\mathrm{E} x_{4}\right| \leq 5.011244 .
\end{aligned}
$$

The numerical solutions, which are stochastic processes, of stochastic dynamo system (3) are obtained by the Euler-Maruyama method. All the stochastic processes' scopes and the ultimate boundary of the corresponding expectations are listed in Table 1. From Table 1, we are pleased to see that the simulation results and the theoretical results of (14) and (15) are consistent.

\section{Globally Exponentially Attractive Set}

Theorem 2. Let $l_{0}=\min \left\{\mu_{1}-(1 / 2) \sigma_{1}^{2}, \mu_{2}-(1 / 2) \sigma_{2}^{2}, \epsilon_{1}-\right.$ $\left.(1 / 2) \sigma_{3}^{2}, \epsilon_{2}-(1 / 2) \sigma_{4}^{2}\right\}$, and $L=\left(L_{1} / l_{0}\right)$. Suppose that the parameters $2 \mu_{1}>\sigma_{1}^{2}, 2 \mu_{2}>\sigma_{2}^{2}, 2 \epsilon_{1}>\sigma_{3}^{2}$, and $2 \epsilon_{2}>\sigma_{4}^{2}$. Then, for any constant $\lambda>0$, the following estimate holds on system (3):

$$
E[V(X)-L] \leq\left[V\left(X_{0}\right)-L\right] \exp \left\{-l_{0}\left(t-t_{0}\right)\right\}
$$

In particular,

$$
\Omega=\{X \mid E V(X) \leq 2 L\}=\left\{X \mid E\left[x_{1}^{2}+\lambda x_{2}^{2}+x_{3}^{2}+\lambda x_{4}^{2}\right] \leq 2 L\right\}
$$

is a globally exponential attractive set of system (3), where

$$
\begin{aligned}
V(X) & =\frac{1}{2}\left(x_{1}^{2}+\lambda x_{2}^{2}+x_{3}^{2}+\lambda x_{4}^{2}\right), \\
L_{1} & =\frac{\left(q_{1}+q_{10} \sigma_{3}\right)^{2}}{2\left(\epsilon_{1}-(1 / 2) \sigma_{3}^{2}\right)}+\lambda \frac{\left(q_{2}+q_{20} \sigma_{4}\right)^{2}}{2\left(\epsilon_{2}-(1 / 2) \sigma_{4}^{2}\right)}+\frac{1}{2} q_{10}^{2}+\frac{1}{2} \lambda q_{20}^{2} .
\end{aligned}
$$

Proof. Define the Lyapunov on $\mathbb{R}^{4}$, where

$$
V(X)=\frac{1}{2}\left(x_{1}^{2}+\lambda x_{2}^{2}+x_{3}^{2}+\lambda x_{4}^{2}\right) \text {. }
$$

Applying Itô's formula to (19), one has 

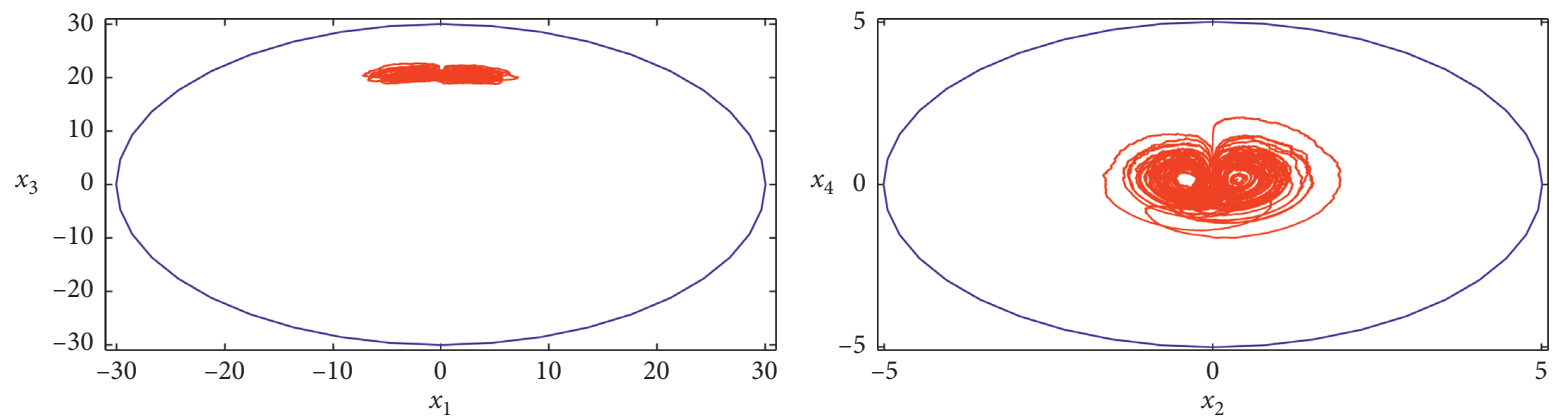

FIGURE 4: The projection of exponentially attractive set of the stochastic dynamo system with $\mu_{1}=3, \mu_{2}=1, \epsilon_{1}=0.1, \epsilon_{2}=0.2, q_{1}=3, q_{2}=1$, $\sigma_{1}=\sigma_{2}=0.1, \sigma_{3}=\sigma_{4}=0.01$, and $q_{10}=q_{20}=0.1$, and initial values $\left(x_{0}, y_{0}, z_{0}, u_{0}\right)=(2.2,2,2.5,3)$.

TABLE 1: Ultimate boundary for stochastic dynamo system.

\begin{tabular}{lcr}
\hline Scopes of stochastic processes & Simulated results of expectation & Theoretical estimates of expectation \\
\hline$-7.692934 \leq x_{1} \leq 7.736921$ & $\mathbf{E} x_{1}=0.104873$ & $\left|\mathbf{E} x_{1}\right| \leq 30.026679$ \\
$-1.484651 \leq x_{2} \leq 2.785851$ & $\mathbf{E} x_{2}=0.033916$ & $\left|\mathbf{E} x_{2}\right| \leq 5.011244$ \\
$1.160790 \leq x_{3} \leq 22.686710$ & $\mathbf{E} x_{3}=19.921792$ & $\left|\mathbf{E} x_{3}\right| \leq 30.026679$ \\
$-1.372995 \leq x_{4} \leq 3.000000$ & $\mathbf{E} x_{4}=0.248853$ & $\left|\mathbf{E} x_{4}\right| \leq 5.011244$ \\
$2.652947 \leq r_{1} \leq 526.916239$ & $\mathbf{E} r_{1}=410.415404$ & $0 \leq \mathbf{E} r_{1} \leq 901.601426$ \\
$0.000000 \leq r_{2} \leq 13.000000$ & $\mathbf{E} r_{2}=0.525692$ & $0 \leq \mathbf{E} r_{2} \leq 25.112567$ \\
\hline
\end{tabular}

$r_{1}=x_{1}^{2}+x_{3}^{2} ; r_{2}=x_{2}^{2}+x_{3}^{2}$.

$\mathrm{d} V(X)=\left[x_{1}\left(-\mu_{1} x_{1}+x_{2} x_{3}\right)+\lambda x_{2}\left(-\mu_{2} x_{2}+x_{1} x_{4}\right)\right.$

$$
\begin{aligned}
& +x_{3}\left(q_{1}-\epsilon_{1} x_{3}-x_{1} x_{2}\right)+\lambda x_{4}\left(q_{2}-\epsilon_{2} x_{4}-x_{1} x_{2}\right) \\
& +\frac{1}{2}\left[\sigma_{1}^{2} x_{1}^{2}+\lambda \sigma_{2}^{2} x_{2}^{2}+\left(\sigma_{3} x_{3}+q_{10}\right)^{2}\right. \\
& \left.\left.+\lambda\left(\sigma_{4} x_{4}+q_{20}\right)^{2}\right]\right] \mathrm{d} t \\
& +\left(\sigma_{1} x_{1}^{2}+\lambda \sigma_{2} x_{2}^{2}+\sigma_{3} x_{3}^{2}+q_{10} x_{3}\right. \\
& \left.+\lambda \sigma_{4} x_{4}^{2}+\lambda q_{20} x_{4}\right) \mathrm{d} W(t) \\
& =\left[-\left(\mu_{1}-\frac{1}{2} \sigma_{1}^{2}\right) x_{1}^{2}-\lambda\left(\mu_{2}-\frac{1}{2} \sigma_{2}^{2}\right) x_{2}^{2}-\left(\epsilon_{1}-\frac{1}{2} \sigma_{3}^{2}\right) x_{3}^{2}\right.
\end{aligned}
$$$$
-\lambda\left(\epsilon_{2}-\frac{1}{2} \sigma_{4}^{2}\right) x_{4}^{2}+\left(q_{1}+q_{10} \sigma_{3}\right) x_{3}
$$$$
\left.+\lambda\left(q_{2}+q_{20} \sigma_{4}\right) x_{4}+\frac{1}{2}\left(q_{10}^{2}+\lambda q_{20}^{2}\right)\right] \mathrm{d} t
$$$$
+\left(\sigma_{1} x_{1}^{2}+\lambda \sigma_{2} x_{2}^{2}+\sigma_{3} x_{3}^{2}+q_{10} x_{3}+\lambda \sigma_{4} x_{4}^{2}\right.
$$$$
\left.+\lambda q_{20} x_{4}\right) \mathrm{d} W(t)
$$$$
=\left[-\frac{1}{2}\left(\mu_{1}-\frac{1}{2} \sigma_{1}^{2}\right) x_{1}^{2}-\frac{1}{2} \lambda\left(\mu_{2}-\frac{1}{2} \sigma_{2}^{2}\right) x_{2}^{2}\right.
$$$$
\left.-\frac{1}{2}\left(\epsilon_{1}-\frac{1}{2} \sigma_{3}^{2}\right) x_{3}^{2}-\frac{1}{2} \lambda\left(\epsilon_{2}-\frac{1}{2} \sigma_{4}^{2}\right) x_{4}^{2}+F(X)\right] \mathrm{d} t
$$$$
+\left[2 \sigma_{1} x_{1}^{2}+2 \lambda \sigma_{2} x_{2}^{2}+2 \sigma_{3} x_{3}^{2}+2 \lambda \sigma_{4} x_{4}^{2}+G(X)\right] \mathrm{d} W(t),
$$

where

$$
\begin{aligned}
F(X)= & -\frac{1}{2}\left(\mu_{1}-\frac{1}{2} \sigma_{1}^{2}\right) x_{1}^{2}-\frac{1}{2} \lambda\left(\mu_{2}-\frac{1}{2} \sigma_{2}^{2}\right) x_{2}^{2} \\
& -\frac{1}{2}\left(\epsilon_{1}-\frac{1}{2} \sigma_{3}^{2}\right) x_{3}^{2}-\frac{1}{2} \lambda\left(\epsilon_{2}-\frac{1}{2} \sigma_{4}^{2}\right) x_{4}^{2} \\
& +\left(q_{1}+q_{10} \sigma_{3}\right) x_{3}+\lambda\left(q_{2}+q_{20} \sigma_{4}\right) x_{4} \\
& +\frac{1}{2}\left(q_{10}^{2}+\lambda q_{20}^{2}\right) \\
G(X)= & -\sigma_{1} x_{1}^{2}-\lambda \sigma_{2} x_{2}^{2}-\sigma_{3} x_{3}^{2}+q_{10} x_{3}-\lambda \sigma_{4} x_{4}^{2}+\lambda q_{20} x_{4}
\end{aligned}
$$

Then,

$$
\begin{aligned}
F(X) \leq & \sup _{x \in \mathbb{R}^{4}} F(X)=\frac{\left(q_{1}+q_{10} \sigma_{3}\right)^{2}}{2\left(\epsilon_{1}-(1 / 2) \sigma_{3}^{2}\right)}+\lambda \frac{\left(q_{2}+q_{20} \sigma_{4}\right)^{2}}{2\left(\epsilon_{2}-(1 / 2) \sigma_{4}^{2}\right)} \\
& +\frac{1}{2} q_{10}^{2}+\frac{1}{2} \lambda q_{20}^{2}=L_{1} \\
G(X) \leq & \sup _{x \in \mathbb{R}^{4}} G(X)=\frac{q_{10}^{2}}{4 \sigma_{3}}+\frac{\lambda q_{20}^{2}}{4 \sigma_{4}}=L_{2} .
\end{aligned}
$$

From (22) and (23), we can obtain

$$
\mathrm{d} V(X) \leq\left[-l_{0} V(X)+L_{1}\right] \mathrm{d} t+\left[l_{1} V(X)+L_{2}\right] \mathrm{d} W(t) .
$$




$$
\begin{aligned}
& l_{0}=\min \left\{\mu_{1}-\frac{1}{2} \sigma_{1}^{2}, \mu_{2}-\frac{1}{2} \sigma_{2}^{2}, \mu_{3}-\frac{1}{2} \sigma_{3}^{2}, \mu_{4}-\frac{1}{2} \sigma_{4}^{2}\right\}, \\
& l_{1}=4 \max \left\{\sigma_{1}, \sigma_{2}, \sigma_{3}, \sigma_{4}\right\} .
\end{aligned}
$$

From (24) and the calculating the expectation, one obtains

$$
E V(X) \leq V\left(X_{0}\right)+\int_{t_{0}}^{t}\left[-l_{0} E V(X)+L_{1}\right] \mathrm{d} s .
$$

From above inequality, one can obtain

$$
\begin{aligned}
E V(X) & \leq V\left(X_{0}\right) \exp \left\{-l_{0}\left(t-t_{0}\right)\right\}+L_{1} \int_{t_{0}}^{t} \exp \left\{-l_{0}\left(s-t_{0}\right)\right\} \mathrm{d} s \\
& =V\left(X_{0}\right) \exp \left\{-l_{0}\left(t-t_{0}\right)\right\}+\frac{L_{1}}{l_{0}}\left\{1-\exp \left\{-l_{0}\left(t-t_{0}\right)\right\}\right\} .
\end{aligned}
$$

Let $L=\left(L_{1} / l_{0}\right)$. When $E V(X)-L>0, E V\left(X_{0}\right)-L>0$, the following estimate holds:

$$
E[V(X)-L] \leq\left[V\left(X_{0}\right)-L\right] \exp \left\{-l_{0}\left(t-t_{0}\right)\right\} .
$$

Thus,

$$
\lim _{t \rightarrow \infty} E V(X) \leq L
$$

That is

$$
\Omega=\{X \mid E V(X) \leq 2 L\}=\left\{X \mid E\left[x_{1}^{2}+\lambda x_{2}^{2}+x_{3}^{2}+\lambda x_{4}^{2}\right] \leq 2 L\right\} .
$$

Theorem 3. Let $l_{0}=\min \left\{\mu_{1}-(1 / 2) \sigma_{1}^{2}, \mu_{2}-(1 / 2) \sigma_{2}^{2}\right.$, $\left.\epsilon_{1}-(1 / 2) \sigma_{3}^{2}, \epsilon_{2}-(1 / 2) \sigma_{4}^{2}\right\}$, and $L=\left(L_{\lambda \eta} / l_{0}\right)$. Suppose that the parameters $2 \mu_{1}>\sigma_{1}^{2}, 2 \mu_{2}>\sigma_{2}^{2}, 2 \epsilon_{1}>\sigma_{3}^{2}$, and $2 \epsilon_{2}>\sigma_{4}^{2}$. Then, for any constant $\lambda>0$ and $\eta \in \mathbb{R}$, the following estimate holds on system (3):

$$
E[V(X)-L] \leq\left[V\left(X_{0}\right)-L\right] \exp \left\{-l_{0}\left(t-t_{0}\right)\right\} .
$$

In particular,

$$
\begin{aligned}
\Omega= & \{X \mid E V(X) \leq 2 L\}=\left\{X \mid E\left[x_{1}^{2}+\lambda x_{2}^{2}+\left(x_{3}+\lambda \eta\right)^{2}\right.\right. \\
& \left.+\lambda\left(x_{4}-\eta\right)^{2} \leq 2 L\right\}
\end{aligned}
$$

is a globally exponential attractive set of system (3), where

$$
\begin{aligned}
V(X)= & \frac{1}{2}\left[x_{1}^{2}+\lambda x_{2}^{2}+\left(x_{3}+\lambda \eta\right)^{2}+\lambda\left(x_{4}-\eta\right)^{2}\right] \\
L_{\lambda \eta}= & \frac{\left(q_{1}+q_{10} \sigma_{3}-(1 / 2) \lambda \eta \sigma_{3}^{2}\right)^{2}}{2\left(\epsilon_{1}-(1 / 2) \sigma_{3}^{2}\right)}+\frac{1}{2} \lambda^{2} \eta^{2}\left(\epsilon_{1}-\frac{1}{2} \sigma_{3}^{2}\right) \\
& +\lambda \eta q_{1}+\frac{1}{2} q_{10}^{2} \\
& +\lambda\left[\frac{\left(q_{2}+q_{20} \sigma_{4}-(1 / 2) \eta \sigma_{4}^{2}\right)^{2}}{2\left(\epsilon_{2}-(1 / 2) \sigma_{4}^{2}\right)}+\frac{1}{2} \eta^{2}\left(\epsilon_{2}-\frac{1}{2} \sigma_{4}^{2}\right)\right. \\
& \left.-\eta q_{2}+\frac{1}{2} q_{20}^{2}\right] .
\end{aligned}
$$

Proof. The proof is the same as that for Theorem 2; we omit it here.

Remark 2. Let $\sigma_{1}=\sigma_{2}=\sigma_{3}=\sigma_{4}=q_{10}=q_{20}=0$; then, system (3) is deterministic. Theorem 3 contain the results given in [12] as special cases.

In Theorem 3, let $\mu_{1}=3, \mu_{2}=1, \epsilon_{1}=0.1, \epsilon_{2}=0.2, q_{1}=3$, $q_{2}=1, \sigma_{1}=\sigma_{2}=0.1, \sigma_{3}=\sigma_{4}=0.01, q_{10}=q_{20}=0.1$, and initial values $\left(x_{0}, y_{0}, z_{0}, u_{0}\right)=(2.2,2,10.5,20)$. We give the following estimate of the ultimate boundary:

$$
\Omega=\left\{X \mid \mathbf{E}\left[x_{1}^{2}+x_{2}^{2}+\left(x_{3}+1\right)^{2}+\left(x_{4}-1\right)^{2}\right] \leq 1034.644495\right\} .
$$

This is the globally exponential attractive set and positive invariant set of the stochastic disk dynamo system.

Then, we have the following results of the ultimate boundary about $x_{1}-x_{2}-x_{3}, x_{1}-x_{2}-x_{4}, x_{1}-x_{3}-x_{4}$, and $x_{2}-x_{3}-x_{4}$, which are the exponentially attractive sets of the stochastic disk dynamo system:

$$
\begin{aligned}
\mathbf{E}\left[x_{1}^{2}+x_{2}^{2}+\left(x_{3}+1\right)^{2}\right] & \leq(32.165890)^{2}, \\
\mathbf{E}\left[x_{1}^{2}+x_{2}^{2}+\left(x_{4}-1\right)^{2}\right] & \leq(32.165890)^{2}, \\
\mathbf{E}\left[x_{1}^{2}+x_{2}^{2}+\left(x_{3}+1\right)^{2}+\left(x_{4}-1\right)^{2}\right] & \leq(32.165890)^{2}, \\
\mathbf{E}\left[x_{2}^{2}+\left(x_{3}+1\right)^{2}+\left(x_{4}-1\right)^{2}\right] & \leq(32.165890)^{2} .
\end{aligned}
$$

The numerical solutions, which are stochastic processes, of stochastic dynamo system (3) are obtained by the Euler-Maruyama method. The simulated time series about $x_{1}^{2}+x_{2}^{2}+\left(x_{3}+1\right)^{2}+\left(x_{4}-1\right)^{2}$ is displayed in Figure 5. In addition, the stochastic processes' scopes is

$$
9.887650 \leq x_{1}^{2}+x_{2}^{2}+\left(x_{3}+1\right)^{2}+\left(x_{4}-1\right)^{2} \leq 580.748156
$$

and the corresponding expectation is

$$
\mathbf{E}\left[x_{1}^{2}+x_{2}^{2}+\left(x_{3}+1\right)^{2}+\left(x_{4}-1\right)^{2}\right]=457.015926 .
$$

It is nice to see that the simulation results and the theoretical results of (34) are consistent.

\section{Stochastic Stability}

The purpose of this section is to seek condition for the asymptotic behavior of system (3).

Theorem 4. When perturbed parameters $\sigma_{3}=\sigma_{4}=q_{10}=$ $q_{20}=0$, suppose that the parameters $2 \mu_{1}>\sigma_{1}^{2}$ and $2 \mu_{2}>\sigma_{2}^{2}$. If $\left(q_{1} / \epsilon_{1}\right)+\left(q_{2} / \epsilon_{2}\right)<\sqrt{\left(2 \mu_{1}-\sigma_{1}^{2}\right)\left(2 \mu_{2}-\sigma_{2}^{2}\right)}$, the equilibrium position ( $\left.0,0,\left(q_{1} / \epsilon_{1}\right),\left(q_{2} / \epsilon_{2}\right)\right)$ of system (3) is stochastically asymptotically stable.

Proof. Let $V(X)=(1 / 2)\left(x_{1}^{2}+x_{2}^{2}+\left(x_{3}-\left(q_{1} / \epsilon_{1}\right)\right)^{2}+\right.$ $\left(x_{4}-\left(q_{2} / \epsilon_{2}\right)\right)^{2}$. Then, 


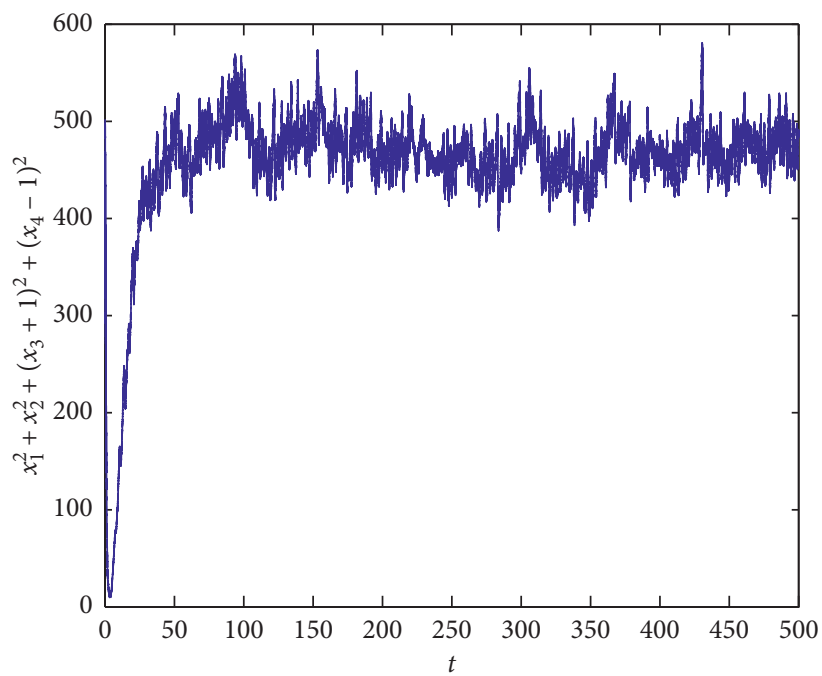

Figure 5: Time series diagrams of the stochastic dynamo system with $u_{1}=0.2, u_{2}=0.5, \epsilon_{1}=0.5, \epsilon_{2}=0.1, q_{1}=5.9, q_{2}=9.15$, $\sigma_{1}=\sigma_{2}=\sigma_{3}=\sigma_{4}=0.1$, and $q_{10}=q_{20}=1$, and initial values $\left(x_{0}, y_{0}, z_{0}, u_{0}\right)=(2.2,2,10.5,20)$.

$$
\begin{aligned}
& L V=x_{1}\left(-\mu_{1} x_{1}+x_{2} x_{3}\right)+x_{2}\left(-\mu_{2} x_{2}+x_{1} x_{4}\right)+\left(x_{3}-\frac{q_{1}}{\epsilon_{1}}\right)\left(q_{1}-\epsilon_{1} x_{3}-x_{1} x_{2}\right) \\
& +\left(x_{4}-\frac{q_{2}}{\epsilon_{2}}\right)\left(q_{2}-\epsilon_{2} x_{4}-x_{1} x_{2}\right)+\frac{1}{2}\left(\sigma_{1}^{2} x_{1}^{2}+\sigma_{2}^{2} x_{2}^{2}\right) \\
& =-\left(\mu_{1}-\frac{1}{2} \sigma_{1}^{2}\right) x_{1}^{2}-\left(\mu_{2}-\frac{1}{2} \sigma_{2}^{2}\right) x_{2}^{2}-\epsilon_{1}\left(x_{3}-\frac{q_{1}}{\epsilon_{1}}\right)^{2}-\epsilon_{2}\left(x_{4}-\frac{q_{2}}{\epsilon_{2}}\right)^{2} \\
& =-\left(x_{1} x_{2} x_{3}-\frac{q_{1}}{\epsilon_{1}} x_{4}-\frac{q_{2}}{\epsilon_{2}}\right)\left(\begin{array}{cccc}
\mu_{1}-\frac{1}{2} \sigma_{1}^{2} & -\frac{1}{2}\left(\frac{q_{1}}{\epsilon_{1}}+\frac{q_{2}}{\epsilon_{2}}\right) & 0 & 0 \\
-\frac{1}{2}\left(\frac{q_{1}}{\epsilon_{1}}+\frac{q_{2}}{\epsilon_{2}}\right) & \mu_{2}-\frac{1}{2} \sigma_{2}^{2} & 0 & 0 \\
0 & 0 & \epsilon_{1} & 0 \\
0 & 0 & 0 & \epsilon_{2}
\end{array}\right)\left(\begin{array}{c}
x_{1} \\
x_{2} \\
x_{3}-\frac{q_{1}}{\epsilon_{1}} \\
x_{4}-\frac{q_{2}}{\epsilon_{2}}
\end{array}\right) \\
& =-\left(x_{1} x_{2} x_{3}-\frac{q_{1}}{\epsilon_{1}} x_{4}-\frac{q_{2}}{\epsilon_{2}}\right) Q\left(\begin{array}{c}
x_{1} \\
x_{2} \\
x_{3}-\frac{q_{1}}{\epsilon_{1}} \\
x_{4}-\frac{q_{2}}{\epsilon_{2}}
\end{array}\right) .
\end{aligned}
$$

When the parameters $2 \mu_{1}>\sigma_{1}^{2}, 2 \mu_{2}>\sigma_{2}^{2}$, and $\left(q_{1} / \epsilon_{1}\right)+\left(q_{2} / \epsilon_{2}\right)<\sqrt{\left(2 \mu_{1}-\sigma_{1}^{2}\right)\left(2 \mu_{2}-\sigma_{2}^{2}\right)}$, the matrix $Q$ is positive-definite. Thus, $L V$ is negative-definite. Then, from
Theorem 4.2.3 of [35], the equilibrium position $\left(0,0,\left(q_{1} / \epsilon_{1}\right),\left(q_{2} / \epsilon_{2}\right)\right)$ of system (3) is stochastically asymptotically stable. 


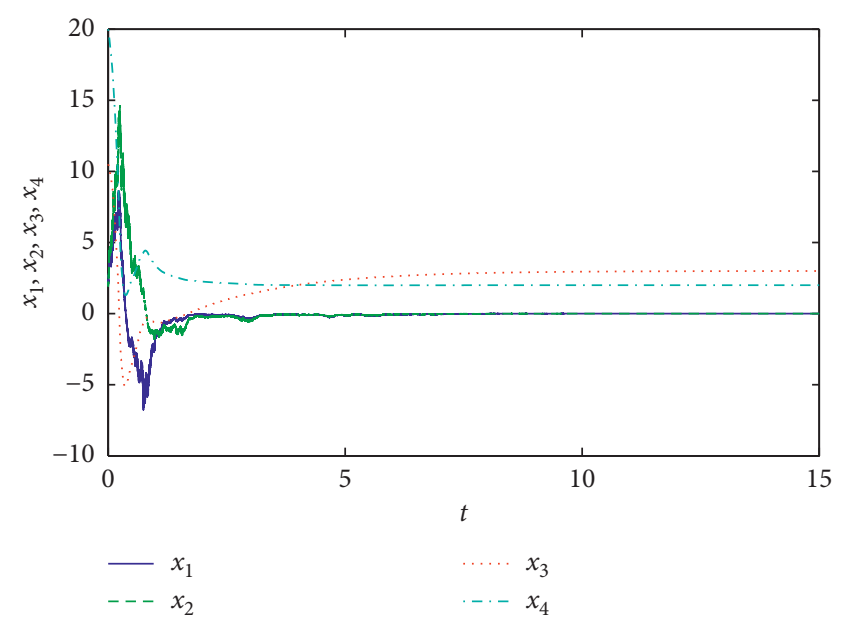

FIGURE 6: Time series diagrams of the stochastic dynamo system with $u_{1}=2.5, u_{2}=2.6, \epsilon_{1}=0.5, \epsilon_{2}=1, q_{1}=1.5, q_{2}=2, \sigma_{1}=1, \sigma_{2}=1$, and $\sigma_{3}=\sigma_{4}=q_{10}=q_{20}=0$, and initial values $\left(x_{0}, y_{0}, z_{0}, u_{0}\right)=(2.2,2$, $10.5,20)$.

Remark 3. When the perturbed parameters $\sigma_{1}=\sigma_{2}=\sigma_{3}=\sigma_{4}=q_{10}=q_{20}=0$, system (3) is deterministic. Suppose $\mu_{1}=\mu_{2}, \epsilon_{1}=\epsilon_{2}$, and $q_{1}=q_{2}$; then, condition $\left(q_{1} / \epsilon_{1}\right)+\left(q_{2} / \epsilon_{2}\right)<\sqrt{\left(2 \mu_{1}-\sigma_{1}^{2}\right)\left(2 \mu_{2}-\sigma_{2}^{2}\right)}$ is reduced to $(q / \epsilon)<\mu$. That is to say, the deterministic disk dynamo system is stable when $(q / \epsilon)<\mu$. That conclusion is coincident with the result of the literature [4].

Remark 4. Comparing the conditions of Theorem 3 and Theorem 4 , the results show that the asymptotically stability of the stochastic disk dynamo system occurs when $\left(q_{1} / \epsilon_{1}\right)+\left(q_{2} / \epsilon_{2}\right)<\sqrt{\left(2 \mu_{1}-\sigma_{1}^{2}\right)\left(2 \mu_{2}-\sigma_{2}^{2}\right)}$, which means the stochastic disk dynamo system will not show chaotic behavior.

Let $u_{1}=2.5, u_{2}=2.6, \epsilon_{1}=0.5, \epsilon_{2}=1, q_{1}=1.5, \quad q_{2}=2$, $\sigma_{1}=1, \sigma_{2}=1$, and $\sigma_{3}=\sigma_{4}=q_{10}=q_{20}=0$, and initial values $\left(x_{0}\right.$, $\left.y_{0}, z_{0}, u_{0}\right)=(2.2,2,10.5,20)$. In Figure 6 , the number results show that the trivial solution of system (3) is stochastically asymptotically stable.

\section{Conclusions}

The coupled dynamo system is a nonlinear dynamical system which is capable of chaotic behaviours. It models the geomagnetic field and is used to explain the reversals in its polarity. Actually, the parameters of the chaotic systems exhibit random fluctuation to a greater or lesser extent, which can carefully describe the disturbance made by environmental noise. The global dynamics of the chaotic disk dynamo system with random fluctuating parameters are concerned, and some new results are presented. Based on the generalized Lyapunov function, the globally attractive and positive invariant set is given, including a two-dimensional parabolic ultimate boundary and a four-dimensional ellipsoidal ultimate boundary. Furthermore, a set of sufficient conditions is derived for all solutions of the stochastic disk dynamo system being global convergent to the equilibrium point. The stochastic disk dynamo system will not show chaotic behavior when the system is stable. Finally, numerical simulations are presented for verification.

\section{Data Availability}

All data generated or analyzed during this study are included in this article.

\section{Conflicts of Interest}

The authors declare that they have no conflicts of interest.

\section{Acknowledgments}

This work was supported by the National Natural Science Foundation of China (Grant nos. 11961074 and 11971414), Natural Science Foundation of Guangxi Province (Grant nos. 2018GXNSFDA281028, 2017GXNSFAA198234, and 2016GXNSFBA380170), the Youth Project of Hunan Provincial Education Department (Grant nos. $18 \mathrm{~B} 518$ and 18B082), the High Level Innovation Team Program from Guangxi Higher Education Institutions of China (Document no. [2018] 35), and the Fundamental Research Funds for the Central Universities, China University of Geosciences (CUGGC05).

\section{References}

[1] G. A. Glatzmaiers and P. H. Roberts, "A three-dimensional self-consistent computer simulation of a geomagnetic field reversal," Nature, vol. 377, no. 6546, pp. 203-209, 1995.

[2] T. Rikitake, "Oscillations of a system of disk dynamos," Mathematical Proceedings of the Cambridge Philosophical Society, vol. 54, no. 1, pp. 89-105, 1958.

[3] A. El-Gohary and R. Yassen, "Adaptive control and synchronization of a coupled dynamo system with uncertain parameters," Chaos, Solitons \& Fractals, vol. 29, no. 5, pp. 1085-1094, 2006.

[4] A. El-Gohary and R. Yassen, "Chaos and optimal control of a coupled dynamo with different time horizons," Chaos, Solitons \& Fractals, vol. 41, no. 2, pp. 698-710, 2009.

[5] E. N. Lorenz, "Deterministic nonperiodic flow," Journal of the Atmospheric Sciences, vol. 20, no. 2, pp. 130-141, 1963.

[6] G. Chen and T. Ueta, "Yet another chaotic attractor," International Journal of Bifurcation and Chaos, vol. 9, no. 7, pp. 1465-1466, 1999.

[7] J. Lü and G. Chen, "A new chaotic attractor coined," International Journal of Bifurcation and Chaos, vol. 12, no. 3, pp. 659-661, 2002.

[8] Q. Yang and G. Chen, "A chaotic system with one saddle and two stable node-foci," International Journal of Bifurcation and Chaos, vol. 18, no. 5, pp. 1393-1414, 2008.

[9] A. Vaněček and S. Čelikovský, Control Systems: From Linear Analysis to Synthesis of Chaos, Prentice-Hall, London, UK, 1996.

[10] S. Čelikovský and G. Chen, "On a generalized Lorenz canonical form of chaotic systems," International Journal of Bifurcation and Chaos, vol. 12, pp. 1789-1812, 2002.

[11] J. Llibre and M. Messias, "Global dynamics of the Rikitake system," Physica D: Nonlinear Phenomena, vol. 238, no. 3, pp. 241-252, 2009. 
[12] J. Jian and Z. Zhao, "New estimations for ultimate boundary and synchronization control for a disk dynamo system," Nonlinear Analysis: Hybrid Systems, vol. 9, pp. 56-66, 2013.

[13] H. Yuan and F. Zhang, "Globally exponentially attractive set of disk dynamo system and its application," Computer Engineering and Applications, vol. 47, no. 33, pp. 226-228, 2011.

[14] H. N. Agiza, "Controlling chaos for the dynamical system of coupled dynamos," Chaos, Solitons \& Fractals, vol. 13, no. 2, pp. 341-352, 2002.

[15] H. N. Agiza, "Chaos synchronization of two coupled dynamos systems with unknown system parameters," International Journal of Modern Physics C, vol. 15, no. 06, pp. 873-883, 2004.

[16] S. Li and Y.-P. Tian, "Global stabilization of a coupled dynamo system," Chaos, Solitons \& Fractals, vol. 16, no. 5, pp. 787-793, 2003.

[17] L. Arnold, Random Dynamical Systems, Springer, New York, NY, USA, 1998.

[18] Z. Huang, J. Cao, and T. Jiang, "Dynamics of stochastic Lorenz-Stenflo system," Nonlinear Dynamics, vol. 78, no. 3, pp. 1739-1754, 2014

[19] Z. Liu, C. Wang, W. Jin, and J. Ma, "Capacitor coupling induces synchronization between neural circuits," Nonlinear Dynamics, vol. 97, no. 4, pp. 2661-2673, 2019.

[20] Z. Yao, J. Ma, Y. Yao, and C. Wang, "Synchronization realization between two nonlinear circuits via an induction coil coupling," Nonlinear Dynamics, vol. 96, no. 1, pp. 205-217, 2019.

[21] Z. Yao, P. Zhou, A. Alsaedi, and J. Ma, "Energy flow-guided synchronization between chaotic circuits," Applied Mathematics and Computation, vol. 374, p. 124998, 2020.

[22] D. Li, X. Wu, and J.-a. Lu, "Estimating the ultimate bound and positively invariant set for the hyperchaotic Lorenz-Haken system," Chaos, Solitons \& Fractals, vol. 39, no. 3, pp. 1290-1296, 2009.

[23] X. Liao, Y. Fu, S. Xie, and P. Yu, "Globally exponentially attractive sets of the family of Lorenz systems," Science in China Series F: Information Sciences, vol. 51, no. 3, pp. 283292, 2008.

[24] P. Yu and X. Liao, "New estimations for globally attractive and positive invariant set of the family of the Lorenz systems," International Journal of Bifurcation and Chaos, vol. 16, no. 11, pp. 3383-3390, 2006.

[25] X. Liao, P. Yu, S. Xie, and Y. Fu, "Study on the global property of the smooth chua's system," International Journal of Bifurcation and Chaos, vol. 16, no. 10, pp. 2815-2841, 2006.

[26] Z. Yan and P. Yu, "Globally exponential hyperchaos (lag) synchronization in a family of modified hyperchaotic rössler systems," International Journal of Bifurcation and Chaos, vol. 17, no. 5, pp. 1759-1774, 2007.

[27] P. Yu and X. Liao, "Globally attractive and positive invariant set of the Lorenz system," International Journal of Bifurcation and Chaos, vol. 16, no. 3, pp. 757-764, 2006.

[28] L. Li, Y. Feng, and Y. Liu, "Dynamics of the stochastic LorenzHaken system," Chaos, Solitons \& Fractals, vol. 91, pp. 670678, 2016.

[29] Y. Liu, Z. Wei, C. Li, A. Liu, and L. Li, "Attractor and bifurcation of forced Lorenz-84 system," International Journal of Geometric Methods in Modern Physics, vol. 16, no. 1, p. 1950002, 2019.

[30] Z. Huang, J. Cao, and T. Jiang, "Dynamics of stochastic Lorenz family of chaotic systems with jump," Journal of Mathematical Chemistry, vol. 52, no. 2, pp. 754-774, 2014.
[31] Y. Liu, L. Li, and X. Wang, "Bifurcation and attractor of the stochastic Rabinovich system with jump," International Journal of Geometric Methods in Modern Physics, vol. 12, no. 09, p. $1550092,2015$.

[32] A. Liu and L. Li, "Global dynamics of the stochastic Rabinovich system," Nonlinear Dynamics, vol. 81, no. 4, pp. 2141-2153, 2015.

[33] Z. Huang, Q. Yang, and J. Cao, "A stochastic model for interactions of hot gases with cloud droplets and raindrops," Nonlinear Analysis: Real World Applications, vol. 12, no. 1, pp. 203-214, 2011.

[34] Z. Huang, Q. Yang, and J. Cao, "Stochastic stability and bifurcation for the chronic state in Marchuk's model with noise," Applied Mathematical Modelling, vol. 35, no. 12, pp. 5842-5855, 2011.

[35] X. Mao, Stochastic Differential Equations and Applications, Horwood Publishing, Chichester, UK, 2nd edition, 2007. 